\title{
Digital Wellness: A Smart Health Care System Using Machine Learning
}

\author{
Anargha P A ${ }^{1}$,Justin Joseph ${ }^{2}$,Sam Sunil ${ }^{3}$,Sanjai $\mathbf{S}^{4}$, Divya Mohan ${ }^{5}$ \\ ${ }^{1}$ Department of Computer Science and Engineering, Albertian Institute of Science and Technology, Kalamassery, \\ Kerala,India,anarghaani1999@gmail.com \\ ${ }^{2}$ Department of Computer Science and Engineering, Albertian Institute of Science and Technology, Kalamassery, \\ Kerala,India,justinmuttathu@gmail.com \\ ${ }^{3}$ Department of Computer Science and Engineering, Albertian Institute of Science and Technology, Kalamassery, \\ Kerala,India, samsunil9061@gmail.com \\ ${ }^{4}$ Department of Computer Science and Engineering, Albertian Institute of Science andTechnology, Kalamassery, \\ Kerala, India,sanjaisubash2000@gmail.com \\ ${ }^{5}$ Assistant Professor, Department of Computer Science and Engineering, Albertian Institute of Science and \\ Technology, Kalamassery, Kerala, India, divyamohan@aisat.ac.in
}

\begin{abstract}
Nowadays, people face various diseases due to environmental condition and their living habits. So the prediction of disease at an earlier stage becomes an important task. But the accurate prediction based on symptoms becomes too difficult for the doctor. The correct prediction of disease is the most challenging task. To overcome this problem data mining plays an important role to predict the disease. Medical science has a large amount of data growth per year. Due to the increasing amount of data growth in the medical and healthcare field the accurate analysis of medical data has been benefits from early patient care. With the help of disease data, data mining finds hidden pattern information in a huge amount of medical data. We proposed general disease prediction based on the symptoms of the patient. For the disease prediction, we use Convolutional neural network (CNN) machine learning algorithm for the accurate prediction of disease. For disease prediction required disease symptoms dataset. After general disease prediction, this system able to gives the risk associated with a general disease which is a lower risk of general disease or higher.
\end{abstract}

Key words: Cross-Validation, Healthcare, Neural network, Online System, Prediction.

\section{INTRODUCTION}

Health is one of the most important assets of our life which directly reflects in any form of progress or development. In today's hustle and bustle of life, most people neglect this asset which may be due to lack of time and complexity in the vast data available over the Internet. Machine Learning has introduced various techniques which would overcome this problem and assist us to emphasize both health and work simultaneously. In the present era, Machine Learning is becoming popular in the healthcare field because there is a need for an efficient analytical methodology for detecting unknown and valuable information in health data. In the health industry, Machine Learning provides several benefits as detection of the fraud in health insurance, availability of medical solution to the patients at lower cost, detection of causes of diseases and identification of medical treatment methods. It also helps the healthcare researchers for making efficient health care policies, constructing drug recommendation systems, developing health profiles of individuals, etc.

In recent years, with the development of computer technologies, the puzzles concerning Bayesian statistics and posterior distribution have been better solved. Meanwhile, Neural Network statistics successfully apply to economic, sociology, and some other fields. In medical fields, foreign scholars have solved some medical problems that are hard to be settled in classic statistics by using Machine Learning Techniques. Without any additional data, classification rules are generated by the training samples themselves. "Smart Health Prediction System" is the computerization of medical information to support and optimize 
Anargha P A et al., International Journal of Advances in Computer Science and Technology, 10(6), June 2021, 5 - 11

(1)Administration of health services

(2) Clinical care

(3) Medical research

(4) Training

It is the application of computing and communication technologies to optimize health information processing by collection, storage, effective retrieval (in due time and place). The proposed system is mainly used by all the people where confidentiality and integrity of the data have the utmost importance. Computer-assisted information retrieval may help support quality decision-making and to avoid human error. Imagine a doctor who has to examine 5 patient records; he or she will go through them with ease. If the number of records grows with a time constraint, it is almost certain that the accuracy with which the doctor delivers the results will not be as high as the ones obtained when he had only five records to be analyzed.

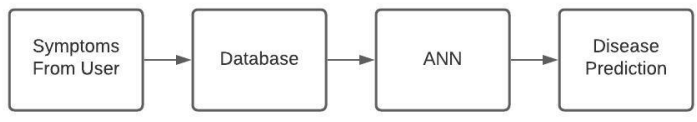

Figure1.Block diagram of Smart HealthCareSystem

\section{LITERATURE SURVEY}

A system that uses the model "Optimized DNN with the usage of Talos" [1] and evaluates the method to others it's far extra efficient than others. This version furnished an excessive accuracy compared to others. In this model, some steps are followed.

Dataset: - It uses the coronary heart illnesses datasets. In heart diseases, 14 attributes are given and 303 columns represent the affected person's statistics. On this database, it is observed that the DNN in the use of Talos and predict the sicknesses.

Data cleaning: - Data cleaning is the primary and essential step for any mission tactics and statistics models. It means filtering and modifying your statistics such that it's far easier to explore and understand.

Feature engineering: -Feature engineering is the most popular part of the deep getting to know. It is used for extracting a few capabilities information sets. It is the process of moving the raw statistics to functions records and these feature records improve the great of the version and offer higher accuracy.

gaining knowledge of algorithms: - we are using the various types of learning algorithms -

1. Logistic Regression

2. CNN

3. Support Vector Machine (SVM)

4. Naïve Bayes
A Machine Learning-based system that predicts common diseases which uses the dataset which contains signs and symptoms was imported from the UCI ML depository [2], in which it contained symptoms of many common illnesses. The machine used $\mathrm{CNN}$ and $\mathrm{KNN}$ as class techniques to gain more than one sickness prediction. Moreover, the proposed answer changed into supplemented with more records that concerned the residing conduct of the tested affected person, which proved to be beneficial in expertise the extent of hazard attached to the predicted disease. The system in comparison with the results between $\mathrm{KNN}$ and $\mathrm{CNN}$ set of rules in phrases of processing time and accuracy. The accuracy and processing time of $\mathrm{CNN}$ were $84.5 \%$ and 11.1 seconds, respectively. The facts proved that the KNN set of rules is underacting as compared to the $\mathrm{CNN}$ set of rules. In mild of this have a look at, the findings additionally agreed that $\mathrm{CNN}$ outperformed usually supervised algorithms which includes KNN, $\mathrm{NB}$, and DT. It concluded that the proposed version scored better in phrases of accuracy, which's explained by using the capability of the version to come across complex nonlinear relationships [2] within the function area. Moreover, CNN detects features with excessive significance that renders a higher description of the ailment, which permits it to correctly expect diseases with excessive complexity.

The proposed system that predicts heart illnesses using supervised ML [3] strategies structured the attributes of statistics as gender, age, chest pain, gender as goal and scope. The ML algorithms that were deployed are DT, KNN, LR, and NB. As per analysis, the LR set of rules gave an excessive accuracy of $86.89 \%$, which is deemed to be the handiest compared to the other mentioned algorithms. The used dataset become imported from the UCI ML laboratory; it turned into comprised one hundred twenty samples that have been coronary heart ailment tremendous, and 150 samples that were heart sickness terrible. The system attempted to assess the overall performance of artificial Neural Networks (ANN), SVM, KNN, NB, LR, and Decision Tree [3]. On the equipment of tenfold pass validation, the results showed that LR has the very best classification accuracy and sensitivity, which suggests excessive dependability at detecting heart illnesses. The studies excelled in undertaking an indepth evaluation of the ML fashions. As an example, various hyper-parameters had been examined at every ML set of rules to converge to the exceptional feasible accuracy and precision values. No matter that advantage, the small length of the imported datasets constraints the getting to know models from concentrated on diseases with better accuracy and precision. 
An expert system called the Smart Health Prediction system [4], which is used for simplifying the task of medical doctors. The system tests a patient at the initial stage and shows the feasible diseases. It starts with asking appropriate symptoms to the patient. If the machine is capable of identifying the right disease, then it suggests a health practitioner be had to the affected person inside the nearest viable area. If the gadget is not certain sufficient, it asks some queries to the patients, still, if the system isn't always sure then it's going to display a few checks to the patient. Primarily based on available cumulative records, the system will display the result.

Merits of the system

1. This causes the disease to be expected more effectively.

2. Furthermore, this proposed gadget also includes various recommendations together with doctor info and prescriptions.

3. There is an expert appointed for each sickness expected. The details of each medical doctor together with their vicinity for every disease could be given.

4. Health practitioner's session price may be prevented at an initial level prescribed drugs are displayed in detail

It is mainly focused on wide scope survey within the subject of device learning knowledge of method in coronary heart ailment. This paper discusses approximately various machine learning sets of rules for heart ailment and their relative comparison at the various parameter. It additionally indicates a future prospectus of machine learning knowledge of algorithms in heart disorder. This paper also does a deep evaluation on the utilization of deep learning [8] knowledge in the area of predicting heart disease.

This paper has summarized the nation of artwork techniques and available strategies for the prediction of this disorder. Deep learning [8] a rising vicinity of artificial intelligence confirmed a few promising results in another field of scientific diagnosis with excessive accuracy. It is nevertheless an open area ready to get applied in heart disorder prediction. Some methods of deep learning have been discussed which can be applied for heart ailment predication, at the side of pioneer device gaining knowledge of algorithms. An analytical contrast has been completed for finding out the best available algorithm for a scientific dataset.

\section{EXISTING METHODOLOGY}

All of us know, we turn into an affected person sometimes or some other and we need good medical care in our existence. We may additionally know doctors are very professional in their career and they may be specialists in specific departments. On every occasion, the scenario isn't the same. For the reason that they're people, the information they collect might not come into suitable effects due to their stress and big work stress. Even though there are big records are needed to examine the remedy, they require a while and know-how to evaluate the statistics. However, the research and exam of the details may additionally beyond the health practitioner's findings. They want a doctor who will communicate to them, see them and advise them inside the remedy within the destiny.

The unfavorable impact of the existing gadget is that the patient needs to go to the physician in-face and didn't get the right remedy considering that they're now not even locating the exact disease. Human errors may be avoided by the usage of the modernday latest computer generation. It is bad while there may be a big amount of information to be classified. Due to stress and tremendous work for people, there may be lower efficiency and accuracy of the decision. Imagine a health practitioner has to check five facts of a patient, he /she can undergo this very easily. when there is a boom in the variety of patient statistics with a time constraint, there'll no longer get a high accuracy one got in comparison to the five statistics he has to check.

\section{DISEASE PREDICTION USING MACHINE LEARNING}

To overcome the drawback of the existing system, we have developed a Smart Healthcare System. Using this system will improve the task of doctors. At the initial stage, the system checks the patient and predicts the possible disease based on the symptoms. The system begins by asking few questions related to the symptoms they feel. If the system was not able to predict the disease or the predicted disease is not sure, then the system will suggest suitable tests related to the symptoms. The system will also provide an option for fixing an appointment with the doctor.

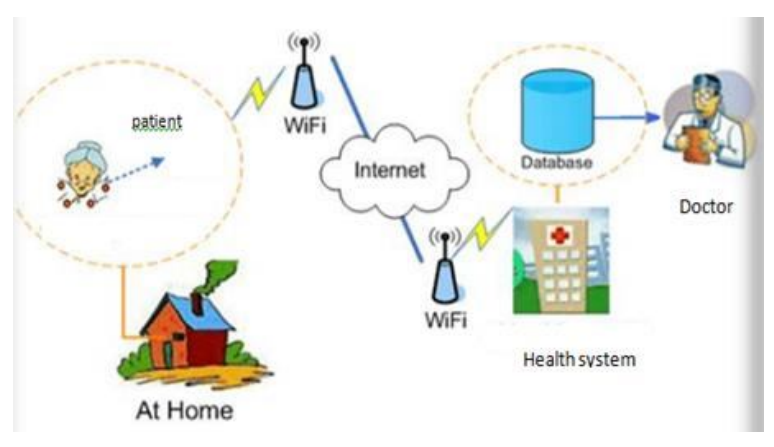

Figure 2.Architecture of Health Prediction using Neural Network Technology 
Anargha P A et al., International Journal of Advances in Computer Science and Technology, 10(6), June 2021, 5 - 11

We've evolved an expert device referred to as Smart Fitness Prediction Machine, which is used for simplifying the task of doctors. A system tests a patient at the initial stage and indicates the feasible illnesses. It begins with asking about symptoms to the patient, if the gadget can discover the ideal ailment, then it indicates a physician to be had to the affected person in the nearest viable area. If the gadget isn't always, positive sufficient, it asks a few queries to the sufferers, still, if the system isn't sure then it's going to show a few tests to the patient. Primarily based on available cumulative facts, the system will show the result. Right here we use a few smart information mining strategies to bet the maximum correct infection that would be associated with the patient's signs and primarily based on the database of several patient's scientific report, an algorithm (Neural Network) is carried out for mapping the signs and symptoms with feasible sicknesses.

\section{WORKING OF MODEL}

Neural networks are typically arranged in layers. Each layer in a layered network is an array of processing elements or neurons. Information flows through each element in an input-output manner. In other words, each element receives an input signal, manipulates it, and forwards an output signal to the other connected elements in the adjacent layer. A common example of such a network is the Multilayer Perceptron (MLP). MLP networks normally have three layers of processing elements with only one hidden layer, but there is no restriction on the number of hidden layers. The only task of the input layer is to receive the external stimuli and to propagate them to the next layer. The hidden layer receives the weighted sum of incoming signals sent by the input units and processes it through an activation function. The activation functions most commonly used are the saturation, sigmoid, and hyperbolic tangent functions.

The hidden units in turn send an output signal towards the neurons in the next layer. This adjacent layer could be either another hidden layer of arranged processing elements or the output layer. The units in the output layer receive the weighted sum of incoming signals and process it using an activation function. Information is propagated forwards until the network produces an output.

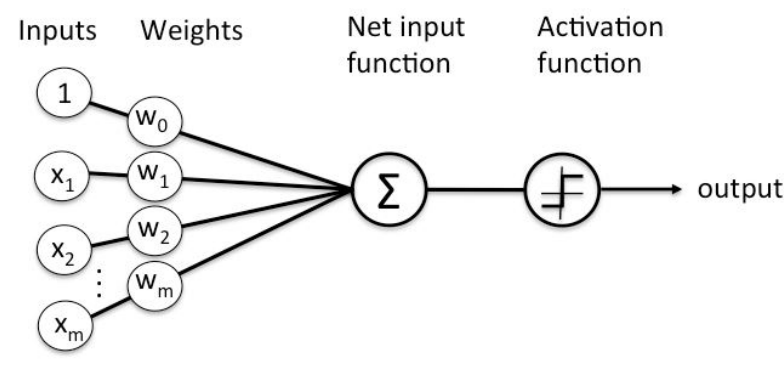

Figure 3.Working of Neural Network Model

For the above general model of artificial neural network, the net input can be calculated as follows -

Yin $=x 1 . w 1+x 2 . w 2+x 3 \cdot w 3 \ldots x m . w m$

i.e., Net input $\mathrm{Yin}=\sum \mathrm{xi}$.wi

The output can be calculated by applying the activation function over the net input.

$\mathrm{Y}=\mathrm{F}(\mathrm{Yin})$

Train and Test Model:The data is split up into training sets and test sets. The training set contains the output field on which the model learns.

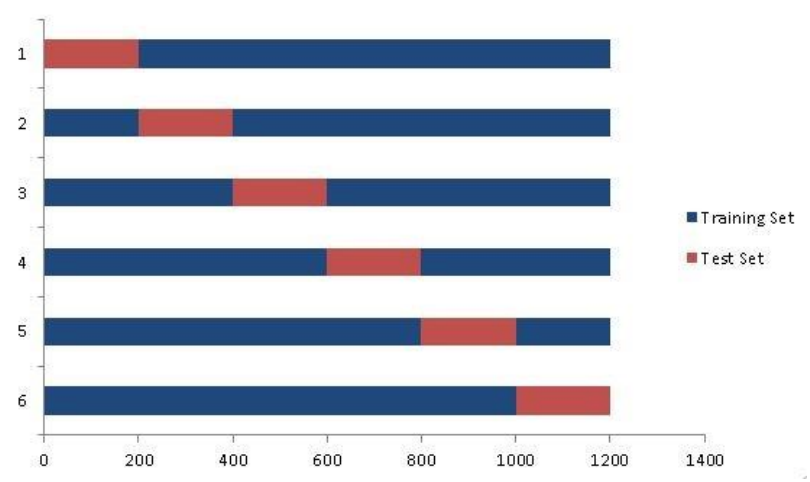

Figure 4.K-FoldCross Validation

Here we use the Cross-Validation method. CrossValidation is broadly speaking utilized in carried out system learning to estimate the skill of a device gaining knowledge of a model on unseen statistics. That is, to use a restricted pattern to estimate how the model is predicted to perform in widespread whilst used to make predictions on information now not used all through the training of the model.

In the training and test models, we are familiarized with two names; Underfitting and Overfitting. An underfit model is not a well suitable model which has very poor performance in the training data set. In the case of overfitting, the performance is poor for the test data set. 
Anargha P A et al., International Journal of Advances in Computer Science and Technology, 10(6), June 2021, 5 - 11
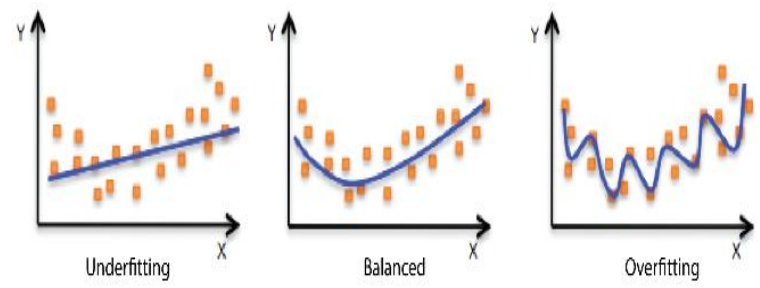

Figure 5.Performance of the system based on Underfitting and Overfitting

\section{RESULT AND ANALYSIS}

Patient Registration: -If affected person is a new user, he will input his non-public information, he will get a user id, and password through which he can login to the device.

Login: - patient Login to the machine using hisid and Password

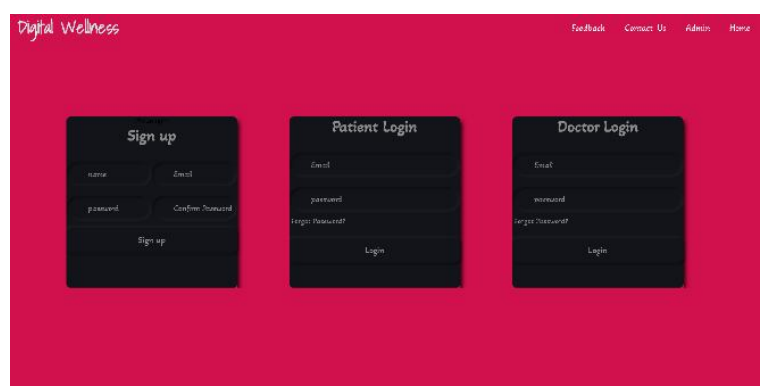

Figure 6.Registration and Sign-up

Disease Prediction: - Affected person will specify the signs prompted because of his contamination. The device will ask certain symptoms concerning his infection after which are expecting the sickness based totally on the signs special by patient.

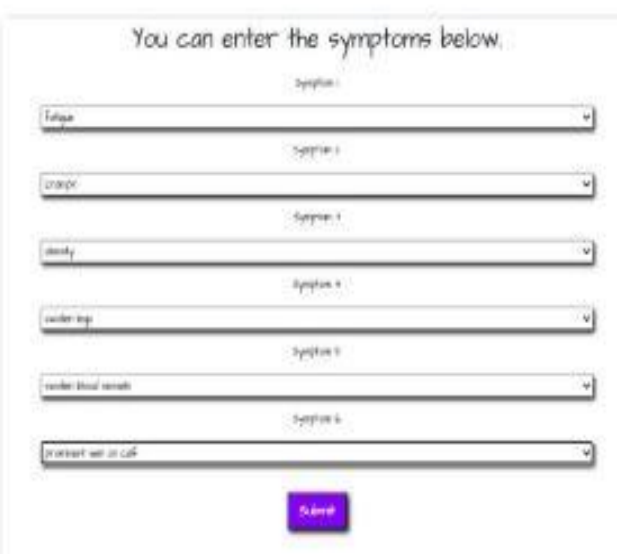

Figure 7.Providing Symptoms

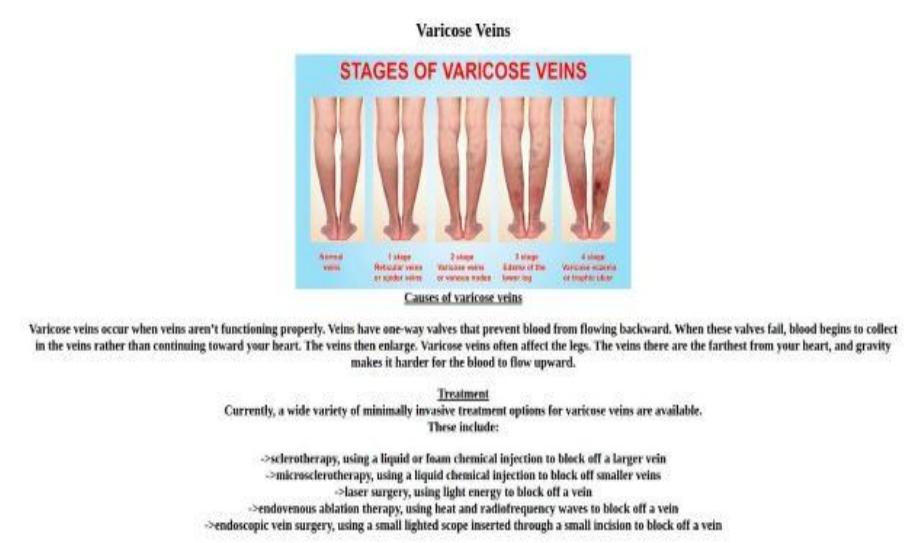

Figure 8.Disease Prediction

Search Doctor: - affected person can look for physician when the disease is predicted with the aid of the device which will provide him with the address of any nearby physician.
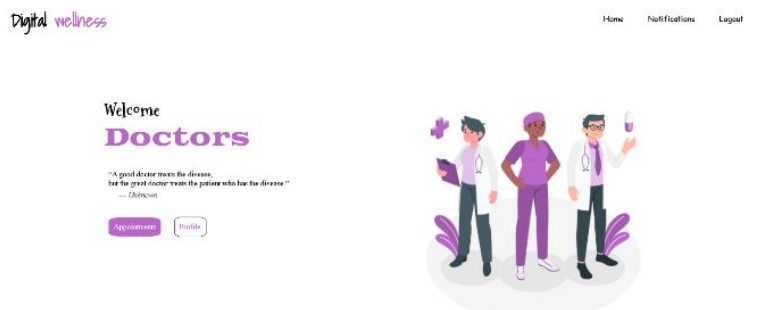

Figure 9. Doctor Login

Get Appointment: -patient will pick an appointment date for the selected doctor.

\section{Technologies Used}

HTML, CSS, JS, BOOTSTRAP: These technologies will be used to create user interface.

PYTHON, PHP: Python is used for connecting various components of User Interface to Database.

MYSQL: MySQL is used as the database at the web server.

Apache HTTP server: In this system we use Apache HTTP server.

The accuracy of the system is $92 \%$. The accuracy of the system is high using the Neural Network algorithm compared to other machine learning algorithms. The system will predict more accurate diseases based on the symptoms provided by the user. 


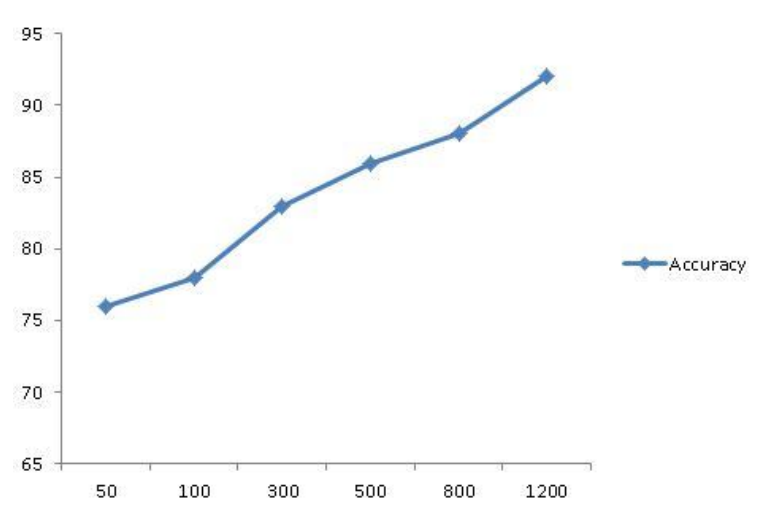

Figure 10.Accuracy Level

Figure 10 shows that accuracy increases with increase in the number of records in the dataset.

\section{CONCLUSION}

Machine Learning can be beneficial in the field of the medical domain. However, privacy, security, and unable to $\log$ into the account are the big problems if they are not addressed and resolved properly. It describes the proposal of a Neural Network model to extract classification knowledge for the aid of various diseases in clinical decision systems and presents a framework of the tool various tools used for analysis. Sometimes the situation occurs when you need the doctor's help immediately, but they are not available due to some reason. In our project, we have designed a new health prediction system, which is an online system, and various patients from any location can view it. Our system comprises of main components such as patient login, enter symptoms in the System, and prescribe medicines, suggested by the nearby doctor. The application takes the input of various symptoms from the patient, analyzes the entered symptoms, and gives appropriate disease prediction. Our system allows the users to get an analysis of the symptoms they give for predicting the disease they are suffering from. Sometimes the situation occurs when you need the doctor's help immediately, but they are not available due to some reason. Thus, it allows the users to get an analysis of the symptoms they give for predicting the disease they are suffering from.

For the future scope, the hidden expertise might be extracted from the historical statistics inside the proposed machine, with the aid of making ready datasets using applying Neural Network algorithm. Predicting smart health can be carried out simplest if gadget responds that manner. Those datasets may be compared with the incoming queries and the final document may be generated by the use of affiliation Rule Mining. Because this proposed technique will work on real ancient statistics, it will offer accurate and green effects, which will help patients, get analysis instantly. greater paintings may be accomplished in the future through the usage of greater information set related to coronary heart sicknesses and with the aid of using specific facts reduction strategies to enhance the class. For higher accuracy and prediction of heart sicknesses, the datasets to be used should be best orientated and unfastened from outliers, inconsistencies, and missing values. This internet utility may be in addition superior in an Android app. this can be available to users on cell foundation and its use may be in addition multiplied. Additionally, characteristics like getting the physician online on chat so that patients can immediately communicate to the doctors. The modules doing cancer analysis may be integrated to locate how near the character associated with most cancers is. This can make this web application predictable in the real sense.

\section{REFERENCES}

[1]Sumit Sharma and Mahesh Parmar, "Heart Diseases Prediction using Deep Learning Neural Network Model", International Journal of Innovative Technology and Exploring Engineering (IJITEE) ISSN: 2278-3075, Volume-9 Issue-3, January 2020

[2]Dhiraj Dahiwade, Prof. Gajanan Patle, Prof. Ektaa Meshram, "Designing Disease Prediction Model Using Machine Learning Approach", Proceedings of the Third International Conference on Computing Methodologies and Communication (ICCMC 2019) IEEE Xplore Part Number: CFP19K25-ART; ISBN: 978-1-5386-7808-4

[3] M.Marimuthu, M. Abinaya, K. S., K. Madhankumar, and V. Pavithra, "A Review on Heart Disease Prediction using Machine Learning and Data Analytics Approach", International Journal of Computer Applications, vol. 181, no. 18, pp. 20-25, 2018

[4] Ambiga.R, Hariraman.S and Anantharaj, "Deep Learning in Smart Health Prediction System", International Research Journal of Latest Trends in Engineering and Technology (IRJLTET), ISSN: 2455-2666, Vol.7, No.1, 2018.

[5]Shraddha Subhash Shirsath "Disease Prediction Using Machine Learning Over Big Data" International Journal of Innovative Research in Science, Vol. 7, Issue 6, June 2018.

[6]Allen Daniel Sunny1, Sajal Kulshreshtha, Satyam Singh3, Srinabh, Mr. Mohan Ba, Dr. Sarojadevi H “ 
Anargha P A et al., International Journal of Advances in Computer Science and Technology, 10(6), June 2021, 5 - 11

Disease Diagnosis System By Exploring Machine Learning Algorithms", International Journal of Innovations in Engineering and Technology (IJIET) Volume 10 Issue 2 May 2018.

[7]A. K. Dwivedi, "Performance evaluation of different machine learning techniques for prediction of heart disease," Neural Computing and Applications, vol. 29, no. 10, pp. 685-693, 2018.

[8]Himanshu Sharma and M ARizvi, "Prediction of Heart Disease using Machine Learning Algorithms: A Survey", International Journal on Recent and Innovation Trends in Computing and Communication ISSN: 2321-8169 Volume: 5 Issue: 8. 2017.

[9]M. Chen, Y. Hao, K. Hwang, L. Wang, and L. Wang, "Disease prediction by machine learning over big data from healthcare communities", IEEE Access, vol. 5, no. 1, pp. 8869-8879, 2017.

[10]Alistair E. W. Johnson, Mohammad M. Ghassemi, Shamim Nemati, Katherine E. Niehaus , "Machine Learning and Decision Support in Critical Care ," in Proc.Int. Conf. Commun., May 2016, pp. $1-6$.

[11]Geoffrey E. Hinton and Simon Osindero Department of Computer Science University of Toronto 10 Kings College Road Toronto, "A fast learning algorithm for deep belief nets" IEEE Trans. Med. Imag., vol. 35, no. 5, pp. 1273-1284, May 2016.

[12] Johnson A. E. W, GhassemiM. M., S. Nemati, Niehaus K. E., Clifton D. A., and Clifford G. D., "Web page classification based on a support vector machine using a weighted vote schema," Proc. IEEE, vol. 104, no. 2, pp. 444-466, Feb. 2016.

[13]V. Kirubha and S. M. Priya, "Survey on Data Mining Algorithms in Disease Prediction," vol. 38, no. 3, pp. 124-128, 2016.

[14]Javier Andreu-Perez.J, Poon .C. C. Y, Merrifield R.D, Wong S. T. C, and G. Z. Yang, "Big data for health," IEEE J. Biomed. Health Information,vol. 19, no. 4, pp. 1193-1208, 2015.

[15]Karen Simonyan_\& Andrew Zisserman and Visual Geometry Group, "Very Deep Convolutional Networks for Large-scale Image Recognition," in Proc. IEEE Conf. Compute. Vis. Pattern Recognit., 2015, pp. 427-436. 